\title{
Adenocarcinoma en conducto ileal después de cistectomía por extrofia vesical
}

\author{
Juaneda Castell B, Angerri Feu O, Caffaratti Sfulcini J, Garat Barredo JM, \\ Villavicencio Mavrich $\mathrm{H}$.
}

Servicio de Urología. Fundació Puigvert. Barcelona.

Actas Urol Esp 2008;32(9):937-940

\section{RESUMEN}

\section{ADENOCARCINOMA EN CONDUCTO ILEAL DESPUÉS DE CISTECTOMÍA POR EXTROFIA VESICAL}

La extrofia vesical es una patología congénita poco frecuente. Hace años se recomendó la realización de cistectomía profiláctica por el riesgo de desarrollar carcinoma en la vejiga extrófica.

Presentamos un caso de extrofia vesical tratado con cistectomía y conducto ileal, desarrollando 34 años después, un adenocarcinoma tipo intestinal en el asa del conducto ileal. Éste es el tercer caso de tumor descrito en un conducto ileal construido después de la cistectomía por extrofia vesical.

Palabras clave: Adenocarcinoma en conducto ileal. Extrofia vesical.

\section{ABSTRACT}

ADENOCARCINOMA IN A ILEAL CONDUIT AFTER CYSTECTOMY FOR BLADDER EXSTROPHY

Exstrophy of the urinary bladder is an uncommon congenital anormaly for wich cystectomy was recommended because of a high risk of developing carcinoma.

We report a patient treated by cystectomy and ileal loop diversion, who, 34 years later, developed carcinoma of the ileal loop. It is the third report of a tumor developing in a ileal conduit constructed after cystectomy for bladder exstrophy.

Keywords: Adenocarcima in ileal loop. Exstrhophy of the uurinary bladder.

$L^{a}$ a extrofia vesical es una patología congénita poco frecuente, aparece en uno de cada 50.000 nacimientos y resulta más frecuente en niños. La causa está en un fallo en la migración del mesénquima a la hora de formar la pared anterior del abdomen y tubulizar la vejiga embrionaria.

A la exposición del tracto urinario inferior por falta de desarrollo del cierre abdominal anterior, se asocian otras anomalias: hernia inguinal, separación de las ramas del pubis, alteración del cuello vesical y reflujo vésico-ureteral.

La reparación quirúrgica de esta malformación ha pasado diferentes etapas, incluso en la década de los 70 se preconizó la realización de cistectomía profiláctica en estos pacientes por presentar un riesgo aumentado de desarrollar adenocarcinoma vesical.

\section{CASO CLINICO}

Paciente de 36 años nacido con extrofia vesical al que se realizó cistectomía y conducto ileal a los dos años de edad. A raíz de un episodio de pielonefritis aguda se detectó ectasia renal bilateral y se colocó sonda de nefrostomía, posteriormente fue derivado a nuestro centro para estudio.

A la exploración física se observaba que el estoma estaba estenosado e incluso era imposible cateterizarlo. Se realizó pielografía anterógrada por sonda de nefrostomía: observando ureterohidronefrosis bilateral, sin defectos de replección y presencia de un stop a nivel del propio estoma (Fig. 1).

Con el diagnóstico de estenosis de la urostomía se intervino quirúrgicamente realizando resección del extremo estenótico y creación de una nueva urostomía. 


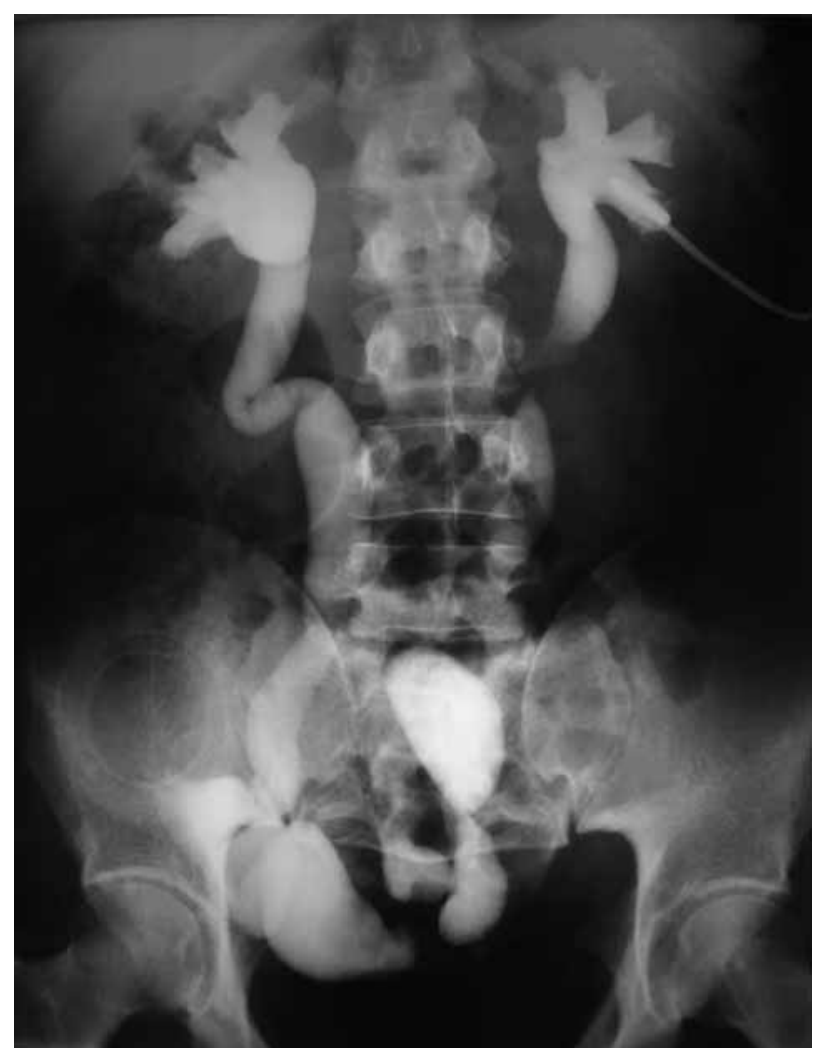

FIGURA 1. Pielografía anterógrada por sonda de nefrostomía: Opacificación de tramo urinario superior y conducto ileal. Stop a nivel de la urostomía.

La anatomía patológica informó de la presencia de un adenocarcinoma tipo ileal que infiltraba todas las capas y los márgenes de resección eran positivos.

El estudio de extensión realizado (ecografía hepática, TAC torácico y abdominal, marcadores tumorales y colonoscopia) solo detectó la presencia de adenopatías localizadas a nivel del conducto ileal (Fig. 2).

En una segunda cirugía se resecó todo el conducto ileal junto a su meso y también la pared abdominal periurostomía, la cual estaba engrosada y fibrótica. Después se configuró un conducto colónico (Fig. 3).

La anatomía patológica del conducto ileal informó de adenocarcinoma tipo intestinal moderadamente diferenciado que infiltraba todas las capas en profundidad y metástasis de adenocarcinoma en dos ganglios del tejido adiposos sub-seroso. Categoría pT3pN1. La pared abdominal resecada también estaba afectada por adenocarcinoma y los márgenes de resección fueron positivos. Actualmente el paciente está realizando quimioterapia (Oxaliplatino+5FU) y radioterapia (Fig. 4).

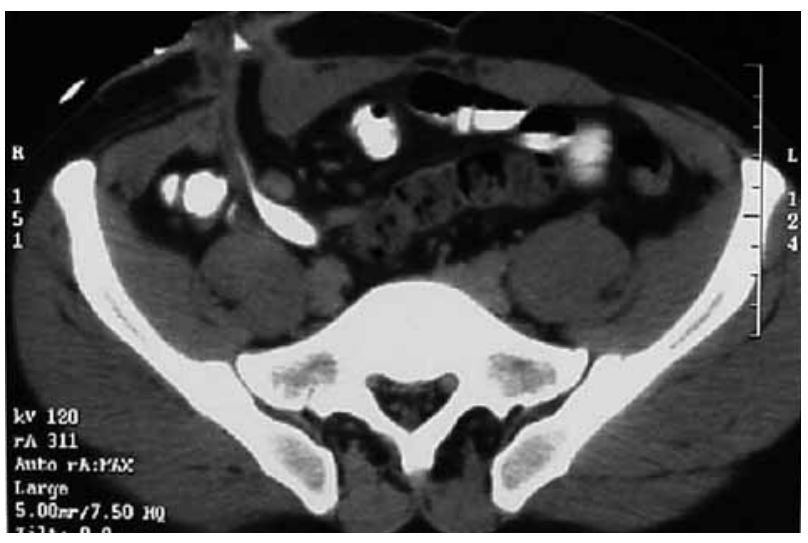

FIGURA 2. TAC abdominal realizado para el estudio de extensión: presencia de adenopatías localizadas a nivel del conducto ileal.

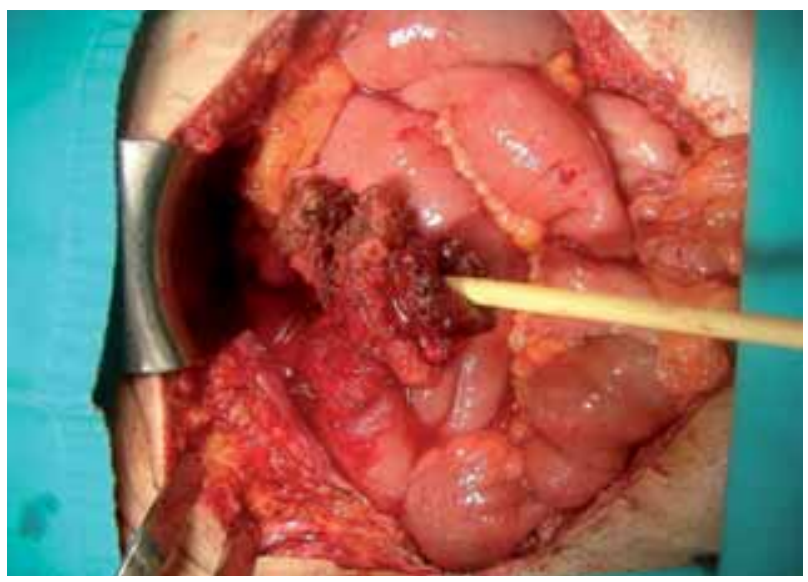

FIGURA 3. Exéresis del conducto ileal.

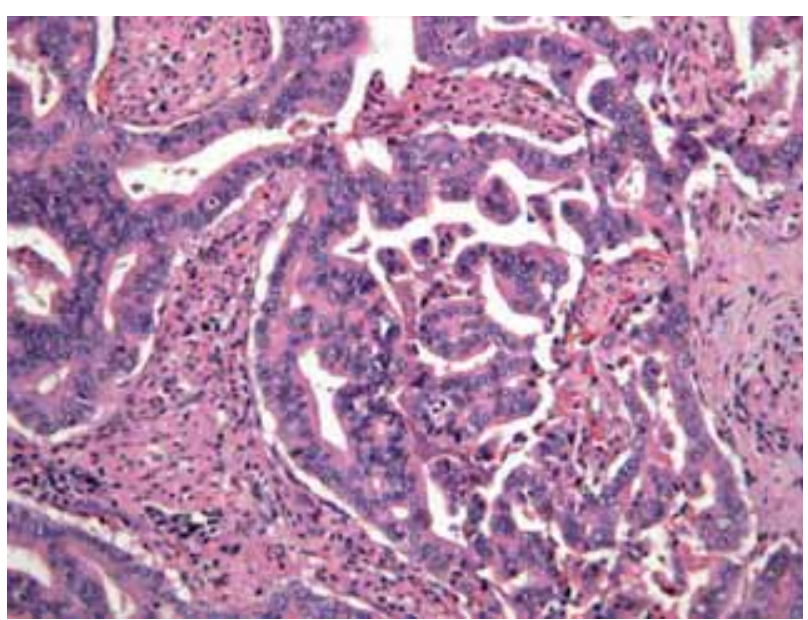

FIGURA 4. Anatomía patológica del conducto ileal: adenocarcinoma tipo intestinal moderadamente diferenciado.

\section{DISCUSION}

Los pacientes nacidos con extrofia vesical presentan un riesgo aumentado de desarrollar adenocarcinoma vesical ${ }^{1}$. 
Mcintosh y Worley consideraron que las constantes infecciones en una vejiga extrófica eran el detonante para la aparición de metaplasia glandular, y junto a la posterior producción de moco, eran la causa del desarrollo de cambios malignos ${ }^{2}$.

Por ello, en la década de los 70 se preconizó la realización de cistectomía profiláctica en estos pacientes debido al riesgo aumentado de desarrollar adecarcinoma vesical. En estos paciente se realizaba la ureterosigmoidostomía o bien un conducto ileal o colónico como forma de derivación urinaria.

Actualmente ya se ha desestimado la realización de la cistectomía profiláctica y se aboga por cierre funcional por etapas: Cierre neonatal de la vejiga, la uretra posterior y la pared abdominal con osteotomía ilíaca en caso de ser necesaria, reparación de las epispadias, y por último la reconstrucción del cuello vesical con procedimiento antirreflujo.

\section{Derivación urinaria y riesgo de neoplasia}

Desde 1920 la ureterosigmoidostomía se ha usado como forma de derivación urinaria ${ }^{3,4}$ pero a partir de 1960 se empezaron a publicar caso de tumores desarrollados en pacientes con ureterosigmoidostomias. Hoy en día sabemos que los pacientes con ureterosigmoidostomía tienen un riesgo de desarrollar adenocarcinoma 500 veces mayor que la población general ${ }^{5}$.

Las teorias de la carcinogénesis incluyen: traumatismo mecánico, irritación crónica, excesiva concentración de electrolitos, bacteriuria y presencia de nitratos ${ }^{6,7}$.

El desarrollo de un tumor en un asa ileal aislada sin estar expuesta al arrastre fecal es extremadamente raro. A los adultos a los que se les realiza la derivación urinaria tipo conducto ileal por patología maligna, en general, no viven el tiempo suficiente para desarrollar neoplasia en el mismo conducto ileal y si la desarrollan, en la mayoría de los casos, es de la misma estirpe que del tumor primario.

La incidencia global del tumor primario de intestino delgado es inferior al $2 \%^{8}$.

En la literatura tan solo hay descritos dos casos de lesiones tumorales a nivel del conducto ileal en pacientes con antecedentes de extrofia vesical.
En 1978 Souza S. describió el primer carcinoma desarrollado 20 años después de la construcción de un conducto ileal en un paciente nacido con extrofia vesical. La anatomía patológica informaba de tumor anaplásico (transicional, escamoso y áreas secretoras de mucina) ${ }^{9}$.

El segundo caso fue publicado por Tomera en 1982: Describió la presencia de un pólipo adenomatoso a nivel proximal del conducto ileal desarrollado 22 años después de la construcción del mismo ${ }^{10}$.

También hay publicado un caso de extrofia vesical tratado con cistectectomía y conducto sigmoide, el cual desarrolló 26 años después, un adenocarcinoma polipoide a nivel de la anastomosis urétero-colónica ${ }^{11}$.

El presente, es el tercer caso descrito de tumor desarrollado en un conducto ileal construido después de la cistectomía por extrofia vesical.

\section{REFERENCIAS}

1. Harzmann R, Schubert GE, Bichler KH. Bladder exstrophy and induction of carcinoma. AKT Urol. 15:116,1984.

2. Mcintosh JF, Worley G Jr. Adenocarcinoma arisisng in exstrophy of the bladder: report of two cases and review of literature. J Urol. 1955;73(5):820-829.

3. Leadbetter GW Jr, Zickerman P, Pierce E. Ureterosigmoidostomy and carcinoma of the colon. J Urol. 1979;121(6):732-735.

4. Filmer RB, Spencer JR. Malignancies in bladder augmnetations and intestinal conduits. J Urol. 1990;143(4):671-678.

5. Chiang MS, Minton JP, Clausen K, Clatworthy HW, Wise HA 2nd. Carcinoma in a colon conduit urinary diversión. J Urol. 1982;127(6): 1185-1187.

6. Stewart M. Urinary diversión and bowel cancer. Ann R Coll Sug Engl. 1986;68(2):98-102.

7. Shokeir AA, Shamaa M, el-Mekresh MM, el-Baz M, Ghoneim MA. Late malignancy in bowel segments exposed to urine without fecal stream. Urology. 1995;46(5):657-661.

8. Perzin KH, Bridge MF. Adenomas of the small intestine: A clinicopathologic review of 51 cases and a study of their relationship to carcinoma. Cancer. 1981;48(3):799-819.

9. Shousha S, Scott J, Polak J. Ileal loop carcinoma alter cystectomy for bladder exstrophy. Br.Med J. 1978;2(6134):397-398.

10. Tomera KM, Unni KK, Utz DC. Adenomatous polyp in ileal conduit. J Urol. 1982;128(5):1025-1026.

11. Chiang MS, Minton JP, Clausen K, Clatworthy HW, Wise HA 2nd. Carcinoma in a colon conduit urinary diversion. J Urol. 1982;127(6): 1185-1187.

Correspondencia autor: Dra. B. Juaneda Castell Servicio de Urología. Fundació Puigvert

Cartagena, 340-350 - 08025 Barcelona. Tel.: 934169700

E-mail autor: bjuaneda73@hotmail.com

Información artículo: Nota clínica

Trabajo recibido: marzo 2007

Trabajo aceptado: abril 2007 


\section{COMENTARIO EDITORIAL}

El caso me parece interesante por cuanto la rareza del mismo es un hecho constatado por lo que un caso más siempre es ilustrativo. Sólo quisiera hacer algunas consideraciones respecto a la ureterosigmoidostomía a la que se hace referencia como contrapunto del Bricker y, especialmente, acentuando la incidencia de tumores en aquellas derivaciones.Estando de acuerdo, en principio, en que hay publicaciones que cifran la mayor incidencia de tumores de colon entre 100 y 500 veces la de la población general, también hay que decir que las hay que lo limitan a 8,5-10,5 veces. Por término medio hay un periodo de latencia en la aparición del tumor de unos 25 años (de 2 a 46 años) y teniendo en cuenta que la máxima supervivencia de una U.S. por cáncer vesical es de 30 años se comprende que no parece decisiva esta circunstancia para evitar la U.S. cuando se interviene por cáncer de vejiga. Ello parece justificar la "vuelta" de algunas escuelas a este tipo de derivación, modificada, como es el Mainz II, en la que la preocupación principal es la disminución de los procesos infecciosos frente a la U.S. clásica.

\section{Referencias}

Ballesteros J.J. y Ballesteros, C.: "Riñón único derivado a sigma: Notable situación morfofuncional a los 51 años de la cirugía”. Arch.Esp.Urol., 56,7 (820-826), 2003.

Dr. J.J. Ballesteros Sampol 